\title{
Reliability Analysis of High Rise Building Considering Wind Load Uncertainty
}

\author{
Yi Zhang ${ }^{a, b}$, Keqin Yan ${ }^{b *}$, Tao Cheng ${ }^{b}$ \\ ${ }^{a}$ Geodetic Institute, Leibniz Universität Hannover, 30167 Germany. \\ ${ }^{b}$ School of Civil Engineering, Hubei Polytechnic University, Huangshi-435003, China.
}

Received 23 January 2018; Accepted 15 March 2018

\begin{abstract}
In engineering structures, the safety problems are always depending on the respond of structures to different types of load. The safety assessment of a high rise building is highly depending on the analysis of environmental load. Many codes and practices have proposed many requirements for engineers in the design works. These include safety factors, limitations on damage, maximum deflections and so on. When violations in these requirements occur, the structure is believed to be dangerous. But once the problem becomes complicated such as multiple unknown loads in one building, it requires reliability analysis in the design. It must take care of all the assumptions and uncertainties in the structural design. In probabilistic assessment, any input variable is considered as an uncertainty. However, the traditional way to deal with these problems may have problems when uncertainties are large. Many probabilistic safety measures need to be reconsidered in engineering work. This paper, we will provide reliability analysis on a high rise building with consideration of wind load. All the most commonly applied reliability methods are been utilized in this analysis and compared base on the performance. The statistical influences including correlation and distribution type are also discussed in the same reliability problem.
\end{abstract}

Keywords: Structural Analysis; Reliability Analysis; Uncertainty Modeling; Wind Engineering.

\section{Introduction}

Wind loading problem is often met in engineering design works especially in the high rise building design. It is a very common natural phenomenon in our real life and is related to quite a lot of loadings to the building structures. In some particular areas such as coastline buildings, it may even suffer hazardous wind load such as hurricanes. This may even create more uncertainty problems in our engineering analysis process.

Many former works have been done on the development of wind related civil engineering research works. Zhang et al. [15] had utilized the concept of copula to model the joint distribution of wind speed and wave height. This concept is then utilized in the structural safety assessment of high rise building and offshore engineering [16-21]. Yan et al. [22] have adopted a stochastic term to characterize the randomness of erosion coefficient when modeling the wind load. This concept was then applied to analyze the stability of buildings when it suffers snow load. Followed the same idea, Cui et al. [27] conducted several experiment studies to investigate the uncertainties regarding the modeling of wind load. For more practical problems in wind related structural analysis, see [5-14]. However, not many works are done on the reliability analysis of high rise building with consideration of wind load [28]. The former works are either only focusing on the reliability methods or emphasizing the practical design code development $[29,30]$. There is a need of full analysis on the existing techniques which are able to perform the structural reliability analysis for high rise building considering wind load. Thus, the significance of the present study is emphasized.

* Corresponding author: 416186133@qq.com

doil http://dx.doi.org/10.28991/cej-0309106

$>$ This is an open access article under the CC-BY license (https://creativecommons.org/licenses/by/4.0/).

(C) Authors retain all copyrights. 
This paper aims to provide a full investigation on the use of all the existing reliability methods for the analysis of high rise building considering wind load. Section 2 will provides a general review of the methods and problems investigated in this study. Section 3 will then presents the problem specification. Detailed analysis is illustrated in Section 4. Section 5 considered a comparison study with numerical calculations. The correlation effect is considered in Section 6. Section 7 analyzes the influences of distribution types to the reliability analysis. The final conclusions are highlighted in Section 8.

\section{Research Methodology}

There are many existing reliability methods in the literature [23-26]. In this paper, the performance of all these fundamental methods are assessed for the problem. This would first start with a crude Monte Carlo simulation study. In performing the Monte Carlo simulation, both Importance Sampling and First Order Reliability Method are tested. The results are compared with integration method. After analyzing the problem using reliability method, the statistical influences including correlation effect and distribution type effect are compared. The results are investigated in view of accuracy. The detailed overview of these methods are illustrated in Figure 1.

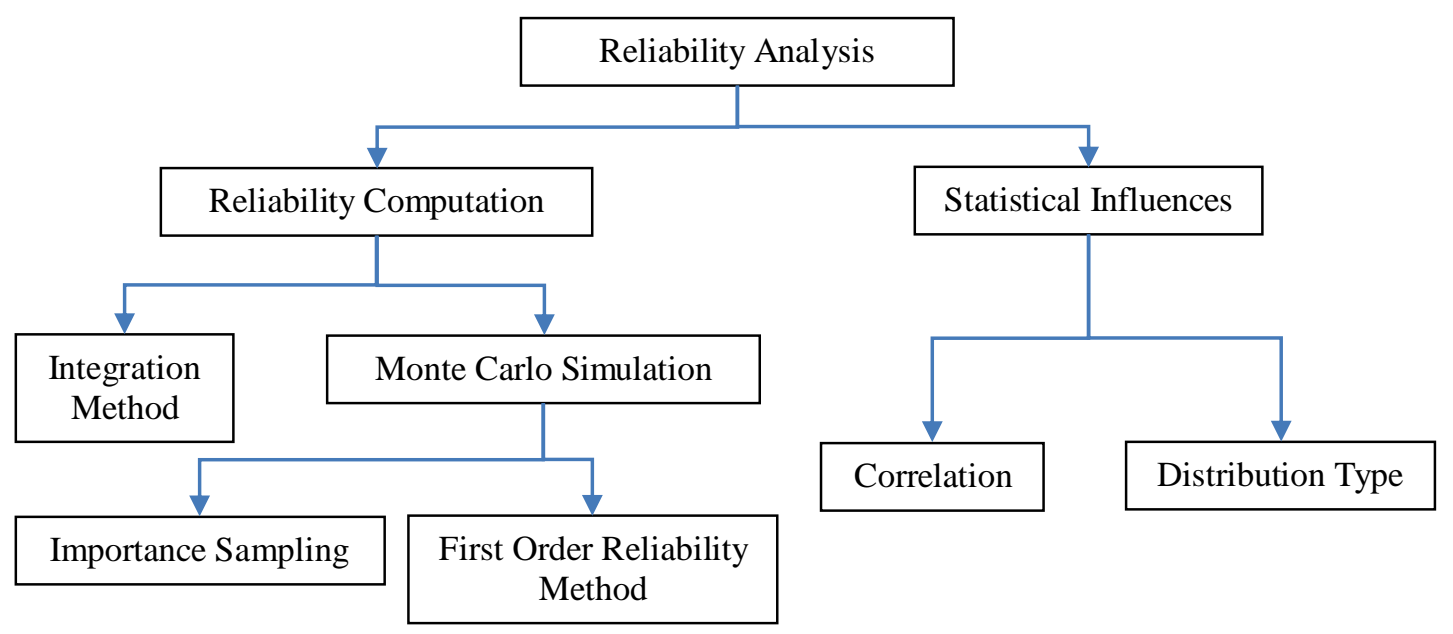

Figure 1. Overview of the methods presented

\section{Problem Specification}

Wind loading problem is very important in the high rise building design. It is a very common natural phenomenon in our real life. In practice, wind loading pressure is always assumed to 'obey' the concept of 'kinetic pressure' q, given by $q(t)=1 / 2 \rho V^{2}(t)$, where the actual pressure onto the building is related to the air density $\rho$ and the time-dependent wind velocity $\mathrm{V}$. The wind force on a simple structure face can thus be written as $P=q A C_{D}^{2}$, in which, $\mathrm{A}$ is the loaded area and $C_{D}$ is the drag coefficient. Catalogue of such coefficients can be found in various design guides and codes [1]. For good application in engineering design works, the basic force formula can be further linearized:

$P(t)=\frac{1}{2} \rho V^{2}(t) A C_{D}=\frac{1}{2} \rho(\bar{V}+u)^{2} A C_{D}=\frac{1}{2} \rho\left(\bar{V}^{2}+2 u \bar{V}\right) A C_{D}$

Where $\bar{V}$ is the mean wind velocity, $u$ is the temporary wind speed acceleration. For more convenient use, we may use the effective loading $\bar{P}=1 / 2 \rho \bar{V}^{2} A C_{D}$ to represent the effect of a real one.

To solve these wind loading problems, many codes and standards have been published. The British, American and European have been highlighted with relevant specifications [2]. Two very famous codes currently used are CP3 and BS6399. Both of these two codes give us the equations to estimate the design wind speed. CP3 has given an equation that the wind speed can be estimated while taking the considerations of topographical, statistical and ground roughness factors [3]. BS6399 has taken the considerations of altitude, direction, seasonal and probability factors [4]. Obviously, we can see that the wind speed is related to quite a lot of uncertainties. In this paper, we are going to do a reliability analysis based on code BS6399 and a simple building prototype in the following:

Determine the deflection of a simple one storey block in building with plan dimension $20 \times 20 \mathrm{~m}$, see Figure 2 . The openings of the building are closed. It is erected in a city at an altitude $100 \mathrm{~m}$ and is $50 \mathrm{~km}$ from the coast. 


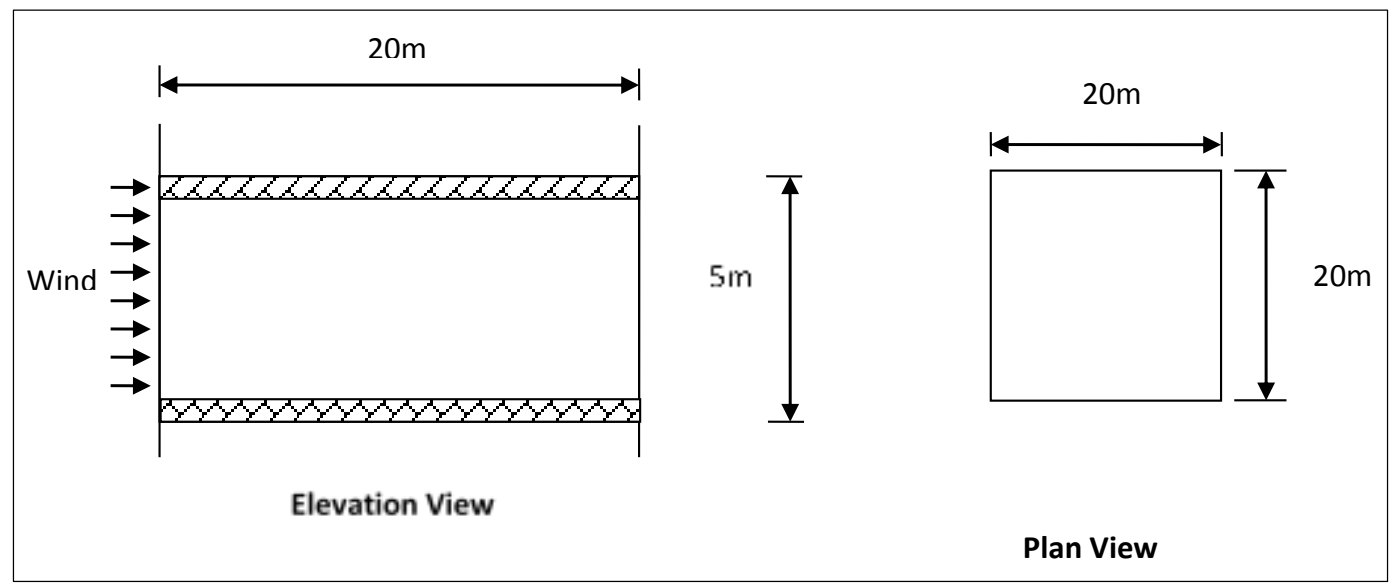

Figure 2. Model view

Based on BS6399, we have the wind speed formula:

$V_{s}=V_{b} \times S_{a} \times S_{d} \times S_{s} \times S_{p}$ and effective wind speed is $V_{e}=S \times V_{s} \times S_{b}$

By applying this load to the building, the deflection can be calculated as:

$\Delta=\frac{\omega l^{4}}{8 E I}=\frac{\left(\frac{1}{2} \rho \bar{V}^{2} C_{D}\right) \times L \times H^{4}}{8 E I}$

Where $\mathrm{L}$ is the length of the structure, $\mathrm{H}$ is the height of the structure, $\mathrm{E}$ is the modulus, I is the moment of inertia. And this is subject to the deflection limit $\frac{\Delta}{H} \leq 0.0025$

Thus, we can write the performance function as:

$G=0.0025-\frac{3 \rho V_{e}^{2} C_{D} H^{4}}{4 B E L^{2}}$

Where $V_{e}$ is the effective wind speed, B is the width of the structure. Some of the coefficients are given in a non-variant value:

The altitude factor: $S_{a}=1+0.001_{\triangle_{S}}=1+0.001 \times 100=1.1$

Drag coefficient: $C_{D}=1.0$

The basic wind load: $V_{b}=38 \mathrm{~m} / \mathrm{s}$

Probability factor: $S_{p}=1.0$

The other factors, such as seasonal and directional factors can be affected by different climate conditions. Thus, assumptions of normal distributions of the following variables have been made, see Table 1 .

Table 1. Parameter information

\begin{tabular}{ccc}
\hline & Mean Value & C.o.V \\
\hline Direction Factor $S_{d}{ }^{2}$ & 0.8 & 0.2 \\
Seasonal Factor $S_{s}{ }^{2}$ & 0.65 & 0.3 \\
Factor $S_{b}{ }^{2}$ & 3.6 & 0.15 \\
Elasticity Modulus $(\mathrm{kN} / \mathrm{m} 2)$ & 200 & 0.2 \\
\hline
\end{tabular}

For solving this problem, we are going to use importance sampling method, gradient projection method, first order second moment method and Monte Carlo simulation in the analysis. Meanwhile, we are going to test the efficiency and accuracy of each method, and also we are looking deep into the effect of distribution type and correlation of the variables to the final result. 


\section{Analysis and Discussion}

\subsection{Monte Carlo Simulation}

In order to see how accuracy each method is, we used the Monte Carlo simulation first to estimate the failure probability. By using 100000 numbers for each uncertain parameter in Minitab, we have obtained a normal-like distribution result for the performance function. The figures are shown as following, see Figure 3.

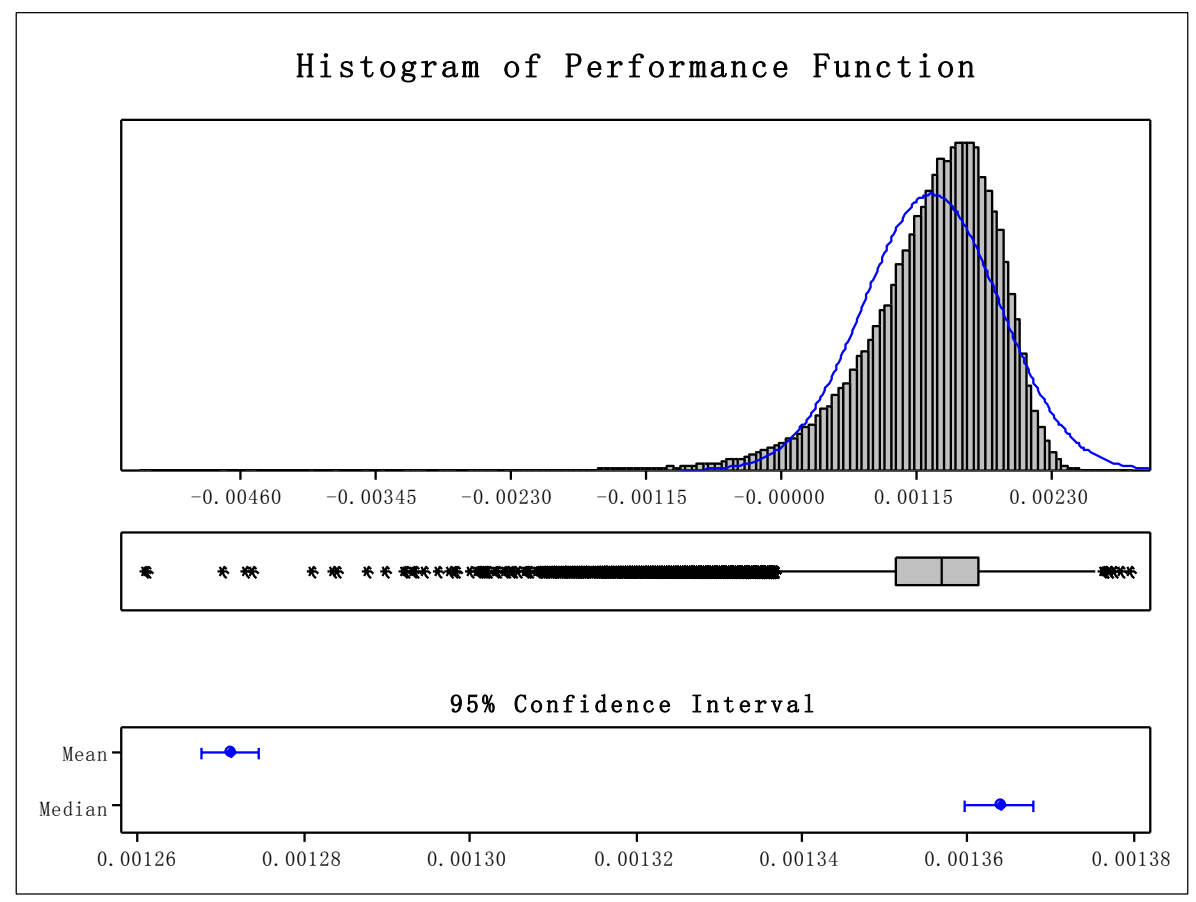

Figure 3. Summary of the Monte Carlo simulation

Since the performance function is a combination of four normal distribution variables, the shape of $\mathrm{G}$ function is quite like a normal one. The mainly difference is the skewness of $\mathrm{G}$ function. And this may be caused by one variable in the denominator. From the output data, we have got a result of $3.43 \%$ failure probability. This result is the most reliable one, since it reflects the real function's pattern in our analysis. But the effort is too much, it needs 100000 calculations, and this calculation may be increased when more variables are present.

Meanwhile, we can do an estimation of the performance equation based on simple calculations:

By using the Taylor's equation:

$\mu_{G} \approx G\left(\overline{S_{s}}, \overline{S_{d}}, \overline{S_{b}}, \bar{E}\right)$

$\sigma_{G}^{2}=\left.\left.\sum_{i=1}^{N} \sum_{j=1}^{N} \frac{\partial G}{\partial X_{i}}\right|_{\overline{X_{i}}} \frac{\partial G}{\partial X_{j}}\right|_{\overline{X_{j}}} \rho \sigma_{i} \sigma_{j}$

And since there is no correlation between each variable, we can get our value based on the non-covariance case:

$\mu_{G} \approx G\left(\overline{S_{s}}, \overline{S_{d}}, \overline{S_{b}}, \bar{E}\right)=0.0025-\frac{3 \rho V_{e}^{2}\left(\overline{S_{s}}, \overline{S_{d}}, \overline{S_{b}}\right) C_{D} H^{4}}{4 B \bar{E} L^{2}}=0.001325$

$\left.\left.\sigma_{G}^{2} \approx \sum_{i=1}^{N} \sum_{j=1}^{N} \frac{\partial G}{\partial X_{i}}\right|_{\overline{X_{i}}} \frac{\partial G}{\partial X_{j}}\right|_{\overline{X_{j}}} \rho \sigma_{i} \sigma_{j}$

$=\left(\frac{\partial G}{\partial S_{b}}\right)^{2} \sigma_{S_{b}}^{2}+\left(\frac{\partial G}{\partial S_{d}}\right)^{2} \sigma_{S d}^{2}+\left(\frac{\partial G}{\partial S_{s}}\right)^{2} \sigma_{S_{s}}^{2}+\left(\frac{\partial G}{\partial E}\right)^{2} \sigma_{E}^{2}=2.65 \times 10^{-7}$

Thus, we could simply obtain the $\beta$ value by $\beta=\mu_{G} / \sigma_{G}$, and the failure probability can be further calculated based on assumed normal distribution: $p=\Phi^{-1}(-\beta)=\Phi^{-1}\left(-\mu_{G} / \sigma_{G}\right)$. The calculated value is $5.044 \times 10^{-3}$ which is smaller than the simulated result. The reason to cause this difference is generally the error occurred in the assumption of normal distribution. So this estimated value is only for reference. 


\subsection{First Order Reliability Method}

In the first order reliability analysis, we often used gradient projection method to locate the critical value $u^{*}$ in the multi-dimensional space. Generally speaking, the method can help us to find the shortest vector which is perpendicular to the limit state function surface. The step improvement is based on each step's gradient in the limit state function. And this method can be used in different distributions by transferring the distribution type to an ordinary normal distribution. Here, in our analysis, we used the Excel to do a iterate operation to find the critical value, so the algorithm is not considered in this project.

By using a constraint of 0.01 to minimize $|G|$ and 0.99 to approximate $\bar{U} \| \vec{G}$, we have got a result of 37.07 for the $U^{T} U$. The failure probability based on this $\beta$ is $5.71 \times 10^{-10}$. And the critical vector $U=\langle-0.64,-0.84,-5.97,0.55\rangle$.

From the result we can see, the FORM can only give us a very fast but rough result. It deviates quite a lot with the actual value in our analysis, since we have a curve performance function. But the good thing is: it can give us the critical vector in the space. This can helps us with the understanding of how much the critical value deviate with the mean value point. And it can be used in a further sampling like the importance sampling method for a further improvement.

\subsection{Importance Sampling}

Importance sampling is often used in the sampling steps while the original probability function can hardly detect the failure value point especially in the multidimensional space. Generally, the original probability function $f(x)$ is very small in the failure domain, and now we can use another probability density function to 'amplify' the effect of some particular area's points. Or in the other words, we can say that the original value is very small and now we use two bigger values multiplied together to calculate this small value. So the choosing of this new probability density function needs to be careful, otherwise, the function may get a very different result, see Figures 4 and 5 .

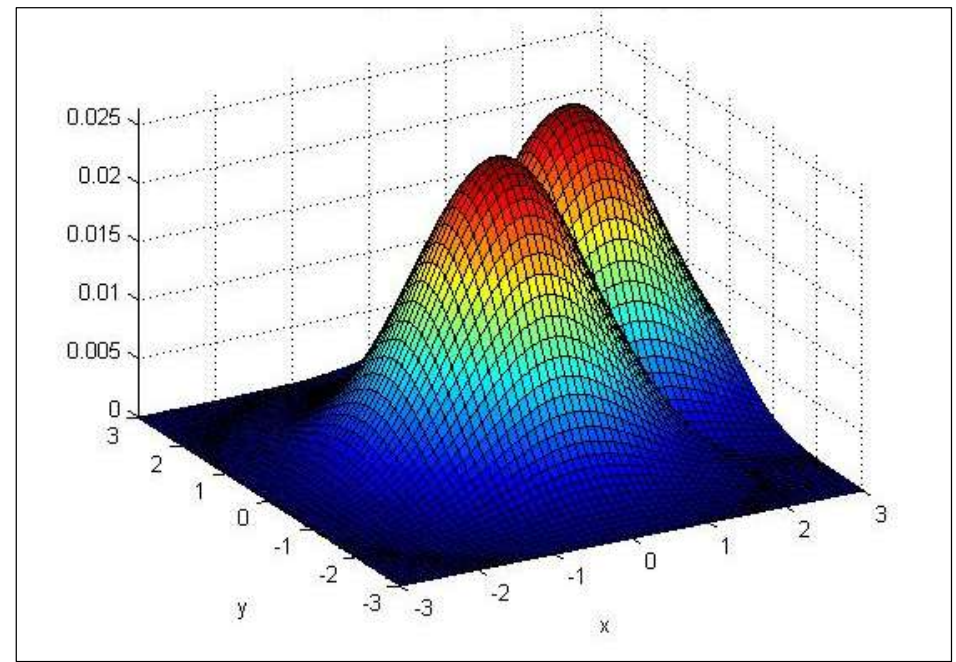

Figure 4. Contour graph of $f$ and g probability density function

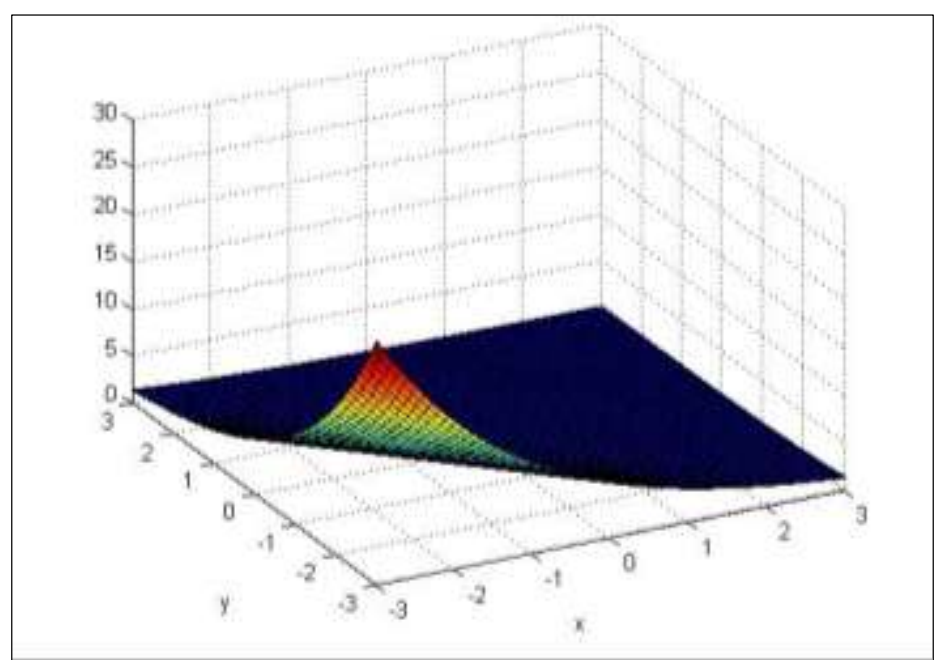

Figure 5. Graph of f/g function value 
Table 2. Parameter distribution

\begin{tabular}{cccc}
\hline$\left\langle u_{1}^{*}, u_{2}^{*}, u_{3}^{*}, u_{4}^{*}\right\rangle$ & Sample Size & $\boldsymbol{\beta}$ Value & Failure Probability \\
\hline$\langle 0.01,0.01,0.01,0.01\rangle$ & 5000 & 1.856 & 0.032 \\
$\langle-0.012,-0.016,-0.12,0.011\rangle$ & 5000 & 1.774 & 0.038 \\
$\langle-0.06,-0.08,-0.6,0.055\rangle$ & 5000 & 1.309 & 0.095 \\
$\langle 0.06,0.08,0.6,-0.055\rangle$ & 5000 & 2.039 & 0.021 \\
\hline
\end{tabular}

$g=\frac{1}{2 \pi} e^{-\frac{1}{2} \pi\left(u_{1}-u_{1}^{*}\right)} \frac{1}{2 \pi} e^{-\frac{1}{2} \pi\left(u_{2}-u_{2}^{*}\right)} \frac{1}{2 \pi} e^{-\frac{1}{2} \pi\left(u_{3}-u_{3}^{*}\right)} \frac{1}{2 \pi} e^{-\frac{1}{2} \pi\left(u_{4}-u_{4}^{*}\right)}$

As a testing first, a joint probability density function is used in the analysis. Initially, we used a very small value to see how the importance sampling changes the final value. The $\left\langle u_{1}^{*}, u_{2}^{*}, u_{3}^{*}, u_{4}^{*}\right\rangle$ is first assigned to $\langle 0.01,0.01,0.01,0.01\rangle$, and the result is very close to the result we get from the Monte Carlo simulation. Then we have used two vectors $\langle-0.012,-0.016,-0.12,0.011\rangle$ and $\langle-0.06,-0.08,-0.6,0.055\rangle$ to calculate the failure probability, see Table 2 . Actually, the proposed function should not affect the output result, but here we have got a larger value. And if we change the value of $\left\langle u_{1}^{*}, u_{2}^{*}, u_{3}^{*}, u_{4}^{*}\right\rangle$ to larger ones, which means the probability density function $\mathrm{g}$ changed to a far point relative to the original point, the result may show a very unreasonable value. This is due to the amplification of some failure points by $\mathrm{g}$ function. Normally speaking, if we have enough sample size, we may not see this problem. But if we only have a small sample size, like here we only have 5000, the result can be easily changed to an unusual value since one failure point in the calculation of $f / g$ can lead to a very large value for the calculation in the failure probability. Actually, before our simulations by using the importance sampling, the value we obtained is already very close to the actual value. It means the failure points are already important in our simulations. There is no need to consider a further importance sampling. Instead, the importance sampling has proposed a biased distribution which 'encouraged' the important values in some specific area. And this creates some error estimating in our sampling. We have tested the importance sampling by centering the probability density function in an opposite direction with the desired vector. And the value drops to a lower value 0.021 which may be dangerous for estimating the limit state.

\section{Numerical Integration}

The most accurate and tedious way to solve a reliability problem is doing the numerical integration for the exact value of the equation. As in this project, the formula is not so sophisticated, we could use a numerical calculation to get the failure probability.

In the numerical integrations, we have used the trapezoidal method, which takes the area of each trapezoidal segment in the division of the probability equation. Because the numerical calculation is a very complicated process, we have used fewer steps to obtain the result. We have given each variable with an interval which can help the computer not to calculate all the values along the whole domain. Then, we could do the integrations along the probability function while the performance function is less than zero. In order to make the calculation more accurate, we tried to make the interval more in the centre and give a finer division to the domain, see Table 3.

Table 3. Factor information

\begin{tabular}{cccc}
\hline Factors & Interval & No. of Divisions & $\begin{array}{c}\text { Breadth of Each } \\
\text { Trapezoidal Area }\end{array}$ \\
\hline Direction Factor $S_{d}{ }^{2}$ & $0.2 \sim 0.4$ & 100 & 0.002 \\
Seasonal Factor $S_{s}{ }^{2}$ & $0.3 \sim 1.0$ & 100 & 0.007 \\
Factor $S_{b}{ }^{2}$ & $2 \sim 6$ & 100 & 0.04 \\
Elasticity Modulus E & $100 \sim 300$ & 100 & 2 \\
\hline
\end{tabular}

The output result for failure probability is 0.0211 , which is very close to the result from Monte Carlo simulation. The difference between these two values may come out from the numerical errors, like the domain is restricted in a smaller one, the breadth of each trapezoid is not small enough, or the round off error may occur in the calculation. A further calculation based on a wider interval has shown a result of 0.0307 , which reduces much error. Anyway, the numerical integration gives us a more reasonable and accurate result. But the effort in the calculations is quite a lot. It may not be used in multiple dimensional reliability analysis. Actually, in this analysis, it already requires $10^{8}$ calculations of the performance function. This is really not an efficient way to estimate the final result. 


\section{Correlation}

Correlation problems can happen very frequently in our engineering problem. It can arise from the dependence between loads or more frequently some assumptions in the factors. Here we can see the wind load direction can always be related to the seasons. Which in a way, it has a correlation effect between these two factors. Thus, we are going to see how the correlation changed the final result in this session.

We assumed some positive correlation coefficients between the factors $S_{s}{ }^{2}$ and $S_{d}{ }^{2}$. Then the C matrix can be used to generate the joint probability and thus the final result can be got from Monte Carlo simulation and numerical calculations, see Figure 6.

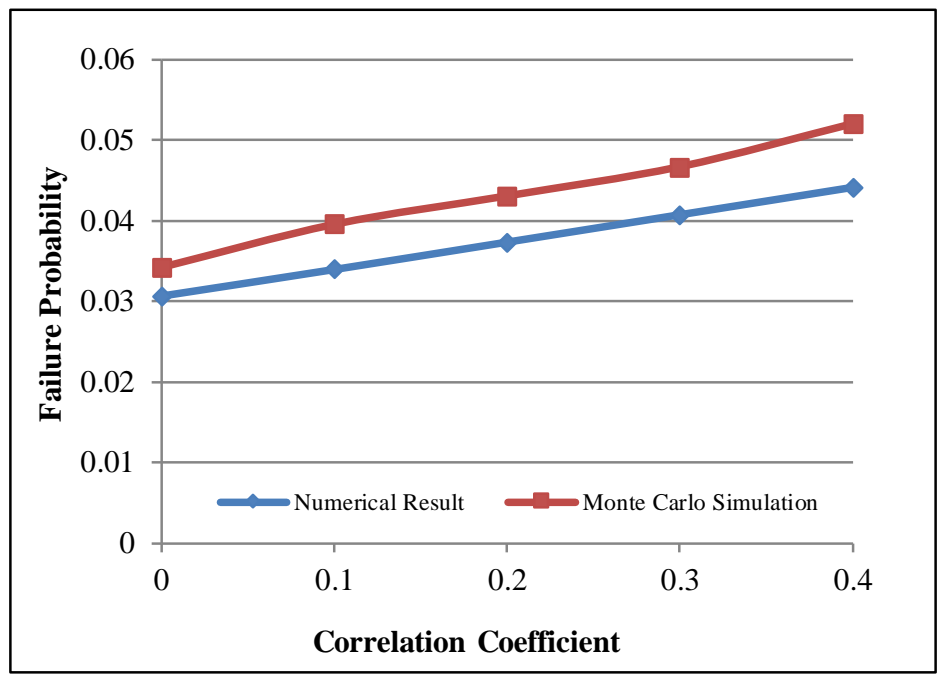

Figure 6. Graph of failure probability with correlation effect

From the compared results from Monte Carlo simulation and numerical integration we can see that the failure probability increases as the correlation becomes severe. The difference between the Monte Carlo simulation and numerical calculation may come from the errors made in the integration calculations. But both method shows good pattern in the increment of failure probability in the way with the increase of correlation coefficient. Besides this correlation, there may be some other correlations, like the negative correlation effect. All of that may create quite different answer. Fortunately, we are only considering the positive correlation effect between these two factors. And it is shown that once this correlation exists, the reliability may be reduced drastically.

\section{Distribution Type}

Besides the correlation effect, we may also meet some different kind of distributions in our analysis. In the FORM analysis, we have changed it to a normal distribution by a transformation. And it will change some formulas in the numerical analysis. The random number generation is changed in the Monte Carlo Simulation for every variable. Nevertheless, the changing is not difficult to manipulate. But the result may deviate quite a lot. This warns us that if there are some wrong assumptions for variables' distributions, it may lead to a final failure in our design. Here we used a lognormal distribution to check how the final result changes with respect to the changing of distribution type, see Table 4.

Table 4. Parameter uncertainty

\begin{tabular}{ccccc}
\hline & Mean Value & C.O.V & Lognormal $\lambda$ & Lognormal $\zeta$ \\
\hline Direction Factor $S_{d}{ }^{2}$ & 0.8 & 0.2 & -0.24275 & 0.198042 \\
Seasonal Factor $S_{s}{ }^{2}$ & 0.65 & 0.3 & -0.47387 & 0.29356 \\
Factor $S_{b}{ }^{2}$ & 3.6 & 0.15 & 1.269809 & 0.149166 \\
Elasticity Modulus $E$ & 200 & 0.2 & 5.278707 & 0.198042 \\
\hline
\end{tabular}

The result from a Matlab programming shows a lower value 0.016 for the failure probability. Even by using a Monte Carlo simulation, this result is still lower than the original normal distribution result. A rough understanding of this result is that the shape of a lognormal distribution may 'concentrate' more at the lower value and thus may results a smaller loading case. But lognormal distribution may be more realistic here, since the value of the factors cannot go to 
negative value. And if we change the distribution to other type, the result will become different again. The distribution of each variable is an assumption, and the calculation of reliability index in highly depending on this, we should be aware of that.

\section{Conclusion}

In this paper, we used a simple wind load problem to investigate and compare the performance of reliability methods. We have compared some common methods by doing the same analysis for this problem. And we also take a further deep view to see how the result can change with the changes of correlation and distribution properties. Some of the key findings are addressed herein:

- In simple reliability problems, Monte Carlo simulation is more suitable as it can give reasonable and accurate result with small statistical uncertainty.

- FORM is not so efficient in a highly nonlinear problem, but it is useful to give a result of the critical vector in a multiple dimensional problem.

- Importance sampling is efficient to estimate small failure probability. But the choosing of the g function may results in large uncertainties, especially if we do not know the critical vector in the multiple spaces.

- Numerical method is an accurate method that can be compare to Monte Carlo simulation. However, the calculation needs quite a lot of efforts. It is not suitable for complicated problems since it requires too much calculation and the numerical error may tend to increase.

- A high positive correlation between the factor Ss and Sd can make the performance function to fail more easily.

- Lognormal distribution assumption can lead the failure probability to a lower value. The assumptions of distribution types for each variable should be taken care of.

\section{Funding}

The work was supported by the National Natural Science Foundation of China [grant number 51478201]; the Innovation Foundation in Youth Science and Technology Team of Hubei Province [grant number T201823]; the Natural Science Fund of Hubei Province [grant number 2012FKC14201]; the Scientific Research Fund of Hubei Provincial Education Department [grant number D20134401]; and the Innovation Foundation in Youth Team of Hubei Polytechnic University [grant number Y0008].

\section{References}

[1] Kappos. A.J. Dynamic Loading and Design of Structures. Spon Press, 2002.doi:10.4324/9780203301951.

[2] Bangash. M.Y.H Prototype Building Structures: Analysis and Design. Thomas Telford Publishing, 1999.doi:10.1680/pbsaad.27770

[3] Basic data for the design of buildings. Chapter V, Wind Loading. CP3, Part2, 1972.doi:10.1201/9781315368221-6.

[4]Loading for buildings. Part 2, Code of practice for wind loads. BS 6399 Part2, 1995.doi:10.3403/02613525.

[5] Melchers. R E.Structural Reliability Analysis and Prediction. John Wiley \& Sons, 1999.doi:10.1002/9781119266105

[8] Zhang Y. and Lam, J. S. L. "Non-conventional modeling of extreme significant wave height through random sets," Acta Oceanologica Sinica, vol. 33, no. 7, pp. 125-130, 2014.doi:10.1007/s13131-014-0508-4.

[9] Zhang, Y., Kim, C.W., Tee, K.F."Maintenance management of offshore structures using Markov process model with random transition probabilities," Structure and Infrastructure Engineering, 13(8), 1068-1080, 2017. doi:10.1080/15732479.2016.1236393.

[10] Zhang Y. and Lam, J. S. L. "Reliability analysis of offshore structures within a time varying environment," Stochastic Environmental Research and Risk Assessment, vol. 29, no. 6, pp. 1615-1636, 2015.doi:10.1007/s00477-015-1084-7.

[11] Smith, J. B. Richels, R. and Miller, B. Potential Consequences of Climate Variability and Change for the Western United States, 2014.doi:10.5860/choice.38-6233.

[12] Ryan, P. C. Stewart, M. G. Spencer, N. and Li, Y. "Probabilistic analysis of climate change impacts on timber power pole networks," International Journal of Electrical Power \& Energy Systems, vol. 78, pp. 513-523, 2016.doi:10.1016/j.ijepes.2015.11.061

[13] Zhang Y. and Lam, J. S. "Estimating economic losses of industry clusters due to port disruptions," Transportation Research Part A: Policy and Practice, vol. 91, pp. 17-33, 2016.doi:10.1016/j.tra.2016.05.017.

[14] Jiang, D. Tian, Z. and Lang, X. "Reliability of climatemodels for China through the IPCC Third to Fifth Assessment Reports," 
International Journal of Climatology, vol. 36, no. 3, pp. 1114-1133, 2016.doi:10.1002/joc.4406.

[15] Zhang, Y. Beer, M. and Quek, S. T. "Long-term performance assessment and design of offshore structures," Computers and Structures, vol. 154, pp. 101-115, 2015.doi:10.1016/j.compstruc.2015.02.029.

[16] Zhang Y. and Lam, J. S. L. “A copula approach in the point estimate method for reliability engineering,” Quality and Reliability Engineering International, vol. 32, pp. 1501-1508, 2016.doi:10.1002/qre.1860.

[17] Zhang Y. and Lam, J. S. L. "Estimating the economic losses of port disruption due to extreme wind events," Ocean \& Coastal Management, vol. 116, pp. 300-310, 2015.doi:10.1016/j.ocecoaman.2015.08.009.

[18] Beer, M. Zhang, Y. Quek, S. T. and Phoon, K. K. "Reliability analysis with scarce information: comparing alternative approaches in a geotechnical engineering context," Structural Safety, vol. 41, pp. 1-10, 2013.doi:10.1016/j.strusafe.2012.10.003.

[19] American Society of Civil Engineers, “Minimum design loads for buildings and other structures," ASCE 7-93, American Society of Civil Engineers, 1993.doi:10.1061/9780784404454.err.

[20] Simiu E. and Scanlan, R. H. Wind Effects on Structures, Wiley- Interscience, 2nd edition, 1986.doi:10.1007/978-3-211-730768 .

[21] Kappos, A. J. Dynamic Loading and Design of Structures, Spon Press, 2002. doi:10.4324/9780203301951.

[22] Yan, K., Cheng, T., Zhang, Y. “A new method in measuring the velocity profile surrounding a fence structure considering snow effects.” Measurement, 116, 373-381, 2018. doi:10.1016/j.measurement.2017.11.032.

[23] Zhang, Y., Kim, C.W., Tee, K.F., Lam, J.S.L. “Optimal sustainable life cycle maintenance strategies for port infrastructures.” Journal of Cleaner Production, 142, 1693-1709, 2017. doi:10.1016/j.jclepro.2016.11.120.

[24] Ramezanzadeh, B., Niroumandrad S, Ahmadi A, Mahdavian M, Moghadam MM. "Enhancement of barrier and corrosion protection performance of an epoxy coating through wet transfer of amino functionalized graphene oxide." Corrosion Science 103 , 283-304, 2016. doi:10.1016/j.corsci.2015.11.033.

[25] Zhang, Y. "Comparing the robustness of offshore structures with marine deteriorations - a fuzzy approach." Advances in Structural Engineering, 18(8), 1159-1172, 2015. doi:10.1260/1369-4332.18.8.1159.

[26] Hirdaris SE, Bai W, Dessi D, Ergin A, Gu X, Hermundstad OA, Huijsmans R, Iijima K, Nielsen UD, Parunov J, Fons eca N. "Loads for use in the design of ships and offshore structures." Ocean engineering. 78:131-74. 2014.doi:10.1016/j.oceaneng.2013.09.012.

[27] Cui, W. and Caracoglia, L. "Examination of experimental variability in HFFB testing of a tall building under multi-directional winds.” Journal of Wind Engineering and Industrial Aerodynamics, 171, 34-49. 2017. doi:10.1016/j.jweia.2017.09.001.

[28] Matta, Fabio, Mabel C. Cuéllar-Azcárate, and Enrico Garbin. "Earthen masonry dwelling structures for extreme wind loads." Engineering Structures 83: 163-175. 2015. doi:10.1016/j.engstruct.2014.10.043.

[29] Patruno, L., M. Ricci, S. de Miranda, and F. Ubertini. "An efficient approach to the evaluation of wind effects on structures based on recorded pressure fields." Engineering Structures 124: 207-220. 2016. doi:10.1016/j.engstruct.2016.06.023.

[30] Zhi, L., Ming F., and Li. Q. S. "Estimation of wind loads on a tall building by an inverse method." Structural Control and Health Monitoring 24,4, 2017. doi:10.1002/stc.1908. 